\title{
Determining responders and non-responders to a whole-grain foods intervention
}

Increased intakes of whole-grain foods such as wholegrain wheat and oats may protect against cardiovascular diseases. In a previous study by our group, participants consumed 3 daily servings of either whole-wheat foods ("wheat", $n=77$ ), 1 serving of whole-wheat and 2 servings of oats ("oats", $n=73$ ), or 3 servings of refined cereal products ("refined", $n=76$ ) as part of their regular diet for 12 weeks after a 4-week run-in period on a refined diet ${ }^{(1)}$. Markers of cardiovascular risk and dietary intake were measured after 6 and 12 weeks intervention. Systolic blood pressure (SBP) significantly decreased $(p<0.05)$ after 12 weeks of intervention in both wholegrain groups compared with the refined group ${ }^{(1)}$. However, there was considerable variation in responsiveness between individuals across outcomes including SBP and cholesterol (Figure 1). This echoes results seen across dietary studies, where typically only $40 \%$ of individuals respond to an intervention ${ }^{(2)}$.

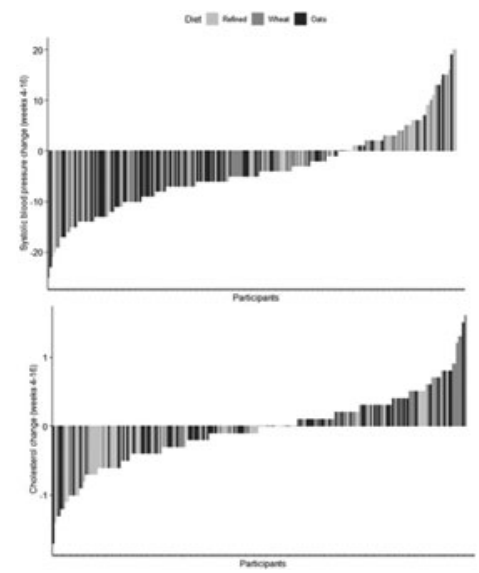

Fig. 1.

We designed and executed an analysis pipeline to consider individual responsiveness to dietary intervention using $\mathrm{R}$ software. This investigated whether (1) baseline markers and measures of dietary intake could predict the blood pressure, cholesterol and triglyceride response to intervention using K-means clustering; and (2) we could define "high" and "low" responders to assess whether both groups could be differentiated based on baseline data. Clusters were compared using one-way ANOVA. Responders and nonresponders were compared using $\mathrm{t}$ tests. Relationships between variables were analysed using simple and multiple linear regression.

K-means clustering on baseline data did not predict the magnitude of response across the outcomes blood pressure, triglycerides, LDL and total cholesterol, despite one cluster having a significantly higher body weight yet lower LDL and total cholesterol levels at baseline. However, we found that participants whose SBP decreased above the median response consumed significantly less dietary cholesterol at baseline than those whose SBP decreased below the median or increased $(p<0.05)$. Those showing a decrease in plasma triglycerides over the course of the study had significantly higher baseline HDL and total cholesterol, but significantly lower pulse pressure than those whose triglycerides increased $(p<0.05)$.

When assessing responsiveness across outcomes, participants that showed a clinically relevant reduction across 3 or more markers had significantly higher baseline LDL, total cholesterol and ApoB levels. Similarly, individuals who showed a reduction in blood pressure at both time points had a significantly greater reduction in blood pressure in comparison to 'inconsistent' response participants. This suggests that individuals at greater risk of cardiovascular disease benefited most from this dietary intervention.

In conclusion, this precision nutrition-style analysis framework has been shown to reveal valuable insights into the main drivers of responsiveness, and may help to evaluate which diet works for whom in future studies ${ }^{(2)}$.

Original study was funded by the Food Standards Agency (Grant NO2035). This work is supported by BBSRC, Unilever and the Scottish Government Rural and Environmental Science and Analytical Services.

1. Tighe P, Duthie G, Vaughan N et al. (2010) Am J Clin Nutr 92, 733-740.

2. de Roos B, Brennan L (2017) Nutrients 9(8), pii: E847. 